\title{
The formation of iron complexes with bile and bile constituents
}

\author{
A. JACOBS AND P. M. MILES \\ From the Department of Haematology, Welsh National School of Medicine, Cardiff
}

SUMMARY Inorganic iron is able to form complexes with whole bile and with some bile constituents and these remain soluble at neutral $p \mathrm{H}$. Ascorbic acid does not appear to play a part in this process. The formation of soluble complexes in vivo is an important factor in maintaining intraluminal iron in a form available for absorption, and bile may have some importance in this respect.

Ionized iron released from food by peptic digestion is in a chemically reactive ferric form. When neutralization occurs, the iron either combines with substances present in solution with it to form complex compounds, or it precipitates as ferric hydroxide. Soluble iron complexes which are stable at neutral or alkaline $p \mathrm{H}$ may be formed with gastric mucopolysaccharide (Jacobs and Miles, 1969a) or with sugars, amino acids, and ascorbic and citric acids (Jacobs and Miles, 1969b). These reactions are essential for the maintenance of intraluminal iron in a form available for absorption. The observations of Conrad and Schade (1968) that ligation of the bile duct in rats impaired iron absorption and their suggestion that iron forms a complex with ascorbic acid in bile stimulated us to investigate the interaction between iron and bile constituents.

The methods used in this study were those described in detail in previous papers, and any minor modifications introduced are described in the individual sections. Iron estimations were carried out by the method of Young and Hicks (1965), and Visking tube (grade 19DC) was used for dialysis. It has been shown (Jacobs and Miles, 1969b) that the concentration of ionizable iron in the stomach after a meal is unlikely to exceed $10^{-4} \mathrm{M}(560 \mu \mathrm{g}$ Fe per $100 \mathrm{ml})$, and this concentration of ferric chloride has been used in the present study.

Received for publication 24 April 1970.

\section{Interaction between Iron and Whole Bile}

When a solution of ferric chloride is mixed with an equal volume of gastric juice before neutralization precipitation of the iron is prevented by the formation of a mucopolysaccharide complex of high molecular weight (Jacobs and Miles, 1969c). A similar experiment has been carried out in which gastric juice was replaced by bile.

Ferric chloride was dissolved in $0.15 \mathrm{M}$ barbitone-acetate buffer (Michaelis) at $p \mathrm{H} 2$ to give an initial concentration of $560 \mu \mathrm{g}$ per 100 $\mathrm{ml}$. This was mixed with an equal volume of a $50 \%$ dilution of bile obtained from a $\mathrm{T}$ tube draining the common bile duct of a patient who had had a cholecystectomy a week previously. The mixture was slowly neutralized by the addition of $12.5 \mathrm{M}$ sodium hydroxide through a finely drawn capillary tube while continuous mixing was maintained with a Fisons Whirlimix. The solution was brought to $p \mathrm{H} 7$ and left to stand for 20 hours. Iron estimation after centrifugation to remove any precipitated matter showed that all the iron remained in solution. The same result was obtained with bile taken directly from normal gallbladders at necropsy and diluted 1:100.

This observation supports the suggestion that an iron-bile complex is formed but gives little information regarding its character. When gel filtration of the iron-bile mixture was carried 
out at $p \mathrm{H} 7$ the iron appeared in the void volume both with Sephadex G-25 and G-200 indicating a large molecular complex. When a ferricascorbate complex was formed in a similar manner using $10^{-2} \mathrm{M}$ ascorbic acid gel filtration with Sephadex G-25 indicated that this was a low molecular weight compound (Fig.). Pretreatment of whole bile with 10 vol hydrogen peroxide at $37^{\circ} \mathrm{C}$ for one hour has no effect on its iron-binding capacity or on the gel filtration characteristics of the complex. Similar treatment resulted in a complete loss of the iron-binding capacity of ascorbic acid. The ascorbic acid concentration in five samples of bile draining from the common bile duct five days after cholecystectomy was estimated by $\mathrm{Dr} R$. E. Hughes and found to be about $10^{-5} \mathrm{M}$. Chelation of $10^{-4} \mathrm{M}$ iron does not occur at this concentration.

It seems improbable that the ascorbic acid content of bile is of primary importance in its ability to bind iron but the large molecular size of the complex formed does not greatly help in characterizing the binding component. Bile has a variable mucus content which could behave in a similar manner to gastric mucus in relation to iron but other components could also be of relevance. Bilirubin itself is known to undergo polymerization at neutral $p \mathrm{H}$ (Broderson and Theilgaard, 1969) and bile salts behave similarly (Haslewood, 1967). These substances are, however, relatively insoluble at $p \mathrm{H} 2$ and are not normally present in the gut in an acid medium.

\section{Interaction between Iron and Bile Components}

An attempt has been made to see whether ferric iron can bind with individual bile components to form complexes soluble at neutral $p \mathrm{H}$. The technique of mixing the potential reactants together at $p \mathrm{H} 2$ followed by neutralization is not effective, as bilirubin and bile salts are insoluble at acid $p \mathrm{H}$, nor does it follow the sequence of events that occurs under physiological conditions. The precipitation of ferric iron at neutral $p \mathrm{H}$ is a time-consuming process, and in a $10^{-4} \mathrm{M}$ solution at $p \mathrm{H} 7$ about half the iron remains soluble for five minutes. If complexing were to occur during that time then precipitation would be prevented, the final concentration of iron remaining in solution depending on the initial rate of precipitation and the rate of complex formation. When no complexing agent is added to the iron solution then the amount remaining in solution in the $p \mathrm{H} \mathrm{6-7}$ range is $18 \%$ at 15 minutes, $11 \%$ at 30 minutes, and virtually nil at one hour.

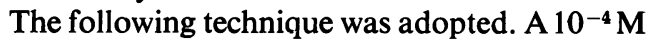
solution of ferric chloride in Michaelis buffer at $p \mathrm{H} 2$ was brought to $p \mathrm{H} 6$ in the usual way and was then immediately mixed with an equal volume of the bile constituent under examination dissolved in water. The $p \mathrm{H}$ of three separate aliquots was adjusted to 6,7 , and 8 respectively, and the mixture allowed to stand for three hours at room temperature. At the end of this time precipitated iron was removed by centrifugation at $1,500 \mathrm{~g}$ for five minutes and the iron concentration in the supernatant solution was measured after acidification. The potential complexing agents added were bile $(50 \%$ dilution of bile from the common bile duct), cholic acid $2 \mathrm{~g}$ per 100 $\mathrm{ml}$, deoxycholic acid $0.2 \mathrm{~g}$ per $100 \mathrm{ml}$, sodium taurocholate $0.2 \mathrm{~g}$ per $100 \mathrm{ml}$, sodium glycocholate $0.8 \mathrm{~g}$ per $100 \mathrm{ml}$, and bilirubin $10 \mathrm{mg}$ per $100 \mathrm{ml}$. The percentage of iron remaining in solution after three hours is shown in the Table.

Whole bile, cholic acid, and sodium glycocholate are all effective in maintaining iron in solution in the $p \mathrm{H}$ range 6-8. Deoxycholic acid

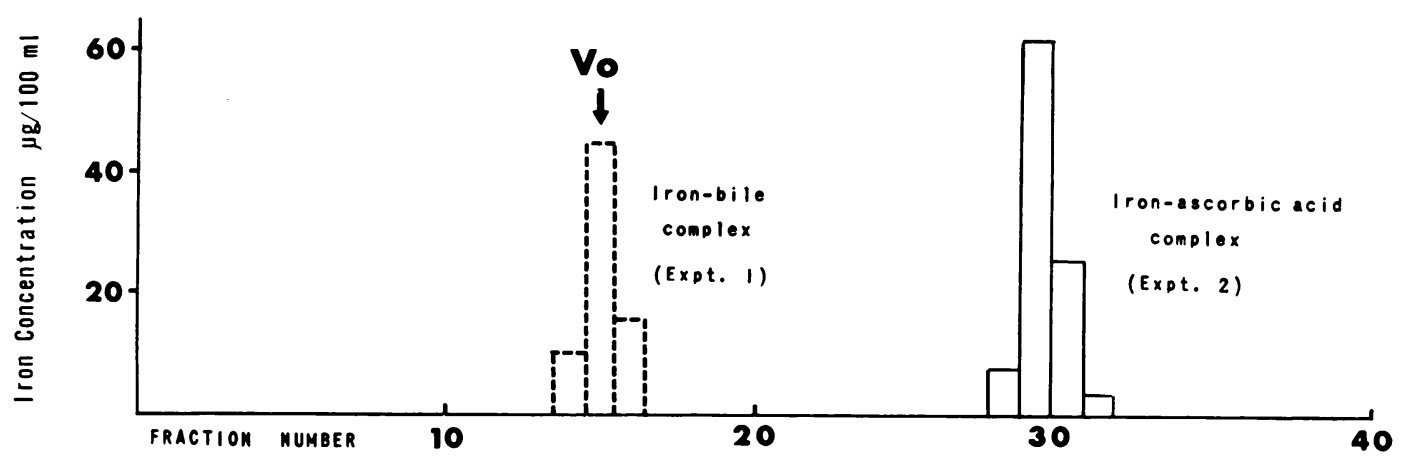

Fig. Gel filtration of iron complexes with whole bile and with ascorbic acid using Sephadex G-25. The results of two separate experiments are shown together. $V_{0}=$ void volume of Sephadex column. 


\begin{tabular}{lrrr}
\hline Substance Added & \multicolumn{3}{l}{ Final $\mathrm{pH}$} \\
\cline { 2 - 4 } & 6 & \multicolumn{1}{c}{7} & \multicolumn{1}{c}{8} \\
\hline Water only & 0 & 0 & 0 \\
Bile & 70 & 73 & 79 \\
Cholic acid $(2 \mathrm{~g} / 100 \mathrm{ml})$ & 75 & 89 & 89 \\
Deoxycholic acid $(0 \cdot 2 \mathrm{~g} / 100 \mathrm{ml})$ & 0 & 45 & 42 \\
Sod. taurocholate $(0 \cdot 2 \mathrm{~g} / 100 \mathrm{ml})$ & 27 & 16 & 18 \\
Sod. glycocholate $(0 \cdot 8 \mathrm{~g} / \mathrm{ml})$ & 90 & 86 & 86 \\
Bilirubin $(10 \mathrm{mg} / 100 \mathrm{ml})$ & 7 & 2 & 41 \\
\hline
\end{tabular}

Table Percentage iron remaining in solution three hours after a solution containing $10^{-4} \mathrm{M}$ ferric iron was neutralized and then mixed with an equal volume of biliary material

and sodium taurocholate are less effective and bilirubin itself is only effective at $p \mathrm{H} 8$, presumably because of its poor solubility in more acid media. When these experiments were carried out with a solution of $10^{-4} \mathrm{M}$ ferrous iron as ferrous sulphate similar results were obtained. Dialysis of the supernatant solutions containing complexed iron against $0.15 \mathrm{M}$ Michaelis buffer for 18 hours at the appropriate $p \mathrm{H}$ showed that all the iron present was non-dialysable. Gel filtration of the iron glycocholate mixture on Sephadex G-200 showed that all the iron appeared in the void volume though some glycocholic acid was retained on the column.

\section{Discussion}

Our results show that when bile is mixed with ionized iron at neutral $p \mathrm{H}$ a complex formation can occur which results in the iron remaining in a soluble form. A similar complex is formed when iron and bile are mixed at acid $p \mathrm{H}$ followed by neutralization. The experiments with ascorbic acid indicate that the iron complex formed has different characteristics from that formed with bile, and when bile is pretreated to destroy any ascorbic acid present complex formation is not affected. It is, therefore, unlikely that this substance is responsible for the iron-binding property of bile as suggested by Conrad and Schade (1968). The high molecular weight of the ironbile complex suggests that it either contains mucoprotein or results from polymerization of a low-molecular weight bile component. Cholic acid and sodium glycocholate have both been shown to form iron complexes, and in these cases also the complex is of a molecular weight in excess of 200,000 . This strongly suggests that complex formation predisposes to polymerization of these substances though it cannot be certain that impurities in the chemicals have not reacted with the iron.

The possible function of iron-bile interaction in vivo is conjectural at the present time. Any mechanism which prevents the precipitation of iron in the gastrointestinal lumen inevitably influences its availability for absorption. The primary site of iron absorption is in the upper jejunum and that of conjugated bile salts in the lower small intestine. It is unlikely that these absorption mechanisms are intimately related. However, the evidence that ligating the bile duct reduces iron absorption (Conrad and Schade, 1968) suggests that bile influences the process, and further studies in vivo are indicated.

\section{References}

Conrad, M. E., and Schade, S. G. (1968). Ascorbic acid chelates in iron absorption: $\mathbf{A}$ role for hydrochloric acid and bile. Gastroenterology, 55, 35-45.

Jacobs, A., and Miles, P. M. (1969a). The iron binding properties of gastric juice. Clin. chim. Acta, 24, 87-92.

Jacobs, A., and Miles, P. M. (1969b). Intraluminal transport of iron from stomach to small-intestinal mucosa. Brit. med. J., 4, 778-781.

Jacobs, A., and Miles, P. M. (1969c). Role of gastric secretion in iron absorption. Gut, 10, 226-229.

Young, D. S., and Hicks, J. M. (1965). Method for the automatic determination of serum iron. J. clin. Path., 18, 98-102.

Brodersen, R., and Theilgaard, J. (1969). Bilirubin colloid formation in neutral aqueous solution. Scand. J. clin. Lab. Invest., 24, 395-398.

Haslewood, G. A. D. (1967). Bile Salts. Methuen, London. 